\title{
DELEUZE Y LA ONTO-TOPOLOGÍA DE LA EXPRESIÓN: EL PLIEGUE COMO MOVIMIENTO FUNDAMENTAL DE LA FILOSOFÍA DE LA DIFERENCIA
}

\author{
Gonzalo Santaya \\ bttps://orcid.org/0000-0002-1998-6215 \\ Consejo Nacional de Investigaciones Científicas y Técnicas \\ Universidad de Buenos Aires \\ https://doi.org/10.15304/ag.40.2.7157
}

\section{Resumen}

Este artículo presenta una interpretación de la ontología desarrollada por Deleuze en Différence et répétition (1968) en clave topológica. Para eso, se argumenta que las tesis deleuzianas relativas al Ser como unívoco y diferencial se apoyan en una teoría de la expresión que supone una concepción de la espacialidad que rebasa la euclidiana. El detalle de esta dinámica espacial es abordado mediante una serie de nociones matemáticas que asisten la producción deleuziana de sus conceptos ontológicos principales, definiendo la expresión y sus momentos a partir de la interacción entre distintos tipos de multiplicidades habitadas por pliegues heterogéneos.

Palabras clave: Deleuze, ontología, topología, expresión, pliegue.

\section{Abstract}

This paper presents an interpretation of the ontology developed by Deleuze in Différence et répétition (1968) under a topological perspective. It maintains that the Deleuzian thesis concerning Being as univocal and differential is supported by a theory of expression which holds a conception of space that goes beyond the Euclidian viewpoint. I will approach

Recibido: 05/10/2020. Aceptado: 09/03/2021. 
this spatial dynamism from a series of mathematical notions that assist the production of Deleuze's main ontological concepts, defining the expression and its moments by the interaction between different types of multiplicities, made of heterogeneous folds.

Keywords: Deleuze, ontology, topology, expression, fold.

\section{Introducción}

Uno de los ejes centrales de la filosofía de Gilles Deleuze, a lo largo de sus diversas etapas de producción, es su recurso a la matemática. Antes que un mero reservorio de metáforas ocasionales, ella traza una línea subterránea que aflora intermitente pero insistentemente en su producción, aportando una clave que ilumina un espíritu general. Así lo indican sus tardías definiciones de la filosofía como una "lógica" o una "teoría de las multiplicidades" (ver Deleuze, 1990, p. 201; y Deleuze y Parnet, 1996, p. $179)^{1}$. Esta noción de multiplicidad, de inspiración riemanniana, subyace a conceptos tan importantes como la Idea-problema (Deleuze, 1968a) o, posteriormente, al espacio liso y al plano de inmanencia (Deleuze y Guattari, 1980 y 1991)². En este trabajo indicaremos cómo el pensamiento deleuziano manifiesta una faceta topológica desde su producción de fines de los 60, que opera sobre el concepto fundamental de expresión. Este concepto no sólo es central en la lectura deleuziana de Spinoza (Deleuze, 1968b), sino también en la elaboración de su propia ontología, expuesta en Diferencia y repetición (1968a). Propondremos un recorrido por esa obra — principalmente sus capítulos IV y V-mostrando cómo las sucesivas fuentes matemáticas empleadas (confluentes en la historia de la topología) ${ }^{3}$ aportan a la determinación del concepto de expresión y sus momentos.

${ }^{1}$ En todos los casos referimos a la paginación de las ediciones originales francesas de Deleuze (ver "Bibliografía"), y las traducciones al español son propias.

${ }^{2}$ Jedrzejewzki (2017) y Plotnitsky (2006) son a nuestro juicio los comentadores que con más rigor han seguido esta pista riemanniana en Deleuze; sin embargo, estudian esta cuestión principalmente en la filosofía del espacio desarrollada en Mil mesetas, dejando en un lugar secundario la ontología de Diferencia y repetición (que pretendemos analizar aquí). Puede verse también Calamari (2015) y Widder (2019) respecto al vínculo DeleuzeRiemann-Bergson.

${ }^{3}$ Desde el cálculo diferencial leibniziano hasta el intuicionismo de Brouwer, pasando por las multiplicidades de Riemann, los grupos de Galois y las singularidades de Poincaré, las referencias de Deleuze (1968a) son hitos ineludibles en toda historia de la topología. Puede consultarse sobre esta historia el anteúltimo capítulo de la monumental obra de Kline (1992, especialmente p. 1536 y ss.). En cuanto a la presencia efectiva de la topología en la obra deleuziana, existen numerosas menciones generales (además de las ya mencionadas, ver Deleuze, 1962, p. 120, y 2002, p. 123, 133, 149, 244), como también referencias puntua- 
Esta propuesta nos obliga, sin embargo, a realizar una aclaración: no intentamos afirmar que la ontología deleuziana está fundada sobre la matemática -afirmación por demás impropia en una filosofía que recusa toda asignación de un fundamento-, ni que haya una equivalencia entre la ontología y una rama de la matemática —como, por ejemplo, afirma Badiou (2007) respecto a la teoría de conjuntos-, pero tampoco una mera relación metafórica entre ambas. El uso que Deleuze hace de la matemática, al igual que el de otras ciencias (física, embriología, geología, etc.) a lo largo de su obra, no apunta ni a un uso literal ni a uno metafórico de las mismas, sino a uno "anexacto". Con esto, Deleuze (1990, p. 45) pretende apelar a nociones científicas "inexactas y sin embargo absolutamente rigurosas", y darles "un rigor que no es directamente científico" —es decir, que no es exacto, numérico, métrico- ${ }^{4}$. En este sentido, la ciencia, el arte y la filosofía, en tanto dominios relativamente autónomos del pensamiento, están sin embargo expuestos a fenómenos de "interferencias" entre ellos, de modo que un filósofo, sin abandonar su campo específico, y en el contexto de un problema concreto, puede tener la necesidad de construir, por ejemplo, "un concepto filosófico propio al espacio riemanniano, o al número irracional" (Deleuze y Guattari, 1991, p. 204). En esta interferencia, se busca extraer ciertos rasgos de una noción matemática para "re-desplegarlos" (Duffy, 2013, pp. 1-3) en el contexto de un problema estrictamente filosófico, y según la especificidad propia de este problema. Intentaremos mostrar que este es el caso respecto las "interferencias" de la topología en la problemática ontológica de Deleuze (1968a).

les a objetos topológicos (así la cinta de Möbius en 1969, p. 31-32) o la noción de pliegue (explícitamente asociada a la topología del pensamiento, en Deleuze, 1986, p. 126; y 1988, pp. 22-23, donde se cita el trabajo del topólogo René Thom). Respecto a los comentadores deleuzianos que recogieron esta línea, quizá la principal sea Burchill (2007), aunque su análisis se ciñe a la topología del spatium y soslaya otros planos de la ontología que abordaremos aquí. El principal comentador de Deleuze y las matemáticas, Duffy (2013), se las ve con esas fuentes de manera fragmentaria y no presenta una interpretación global del pensamiento deleuziano. La obra fundamental de DeLanda (2002, p. 9) señala la centralidad del concepto de multiplicidad en su carácter matemático, pero se vuelca a una descripción más bien cientificista de los sistemas dinámicos, y evita el comentario estrictamente ontológico. Por nuestra parte, en Santaya (2019) exploramos el carácter "topológico" del método de creación conceptual de Deleuze.

${ }^{4}$ Para mayor detalle sobre el concepto de "anexactitud" puede verse Deleuze y Guattari (1980, pp. 454-455). Sobre la relevancia de este enfoque poco ortodoxo de los cruces entre ciencias exactas y filosofía, pueden verse las reflexiones de filósofos de la matemática de la talla de Salanskis (1996) y Zalamea (2020). 
Así como la topología —en términos muy generales- enfoca desde un punto de vista intrínseco (sin apelar a la matriz representativa del espacio euclidiano) el estudio de distintas configuraciones espaciales como fenómenos plásticos, pasibles de variaciones y transformaciones, definiendo dichas configuraciones por la estructura de interconexión de sus puntos o regiones y no por su forma y tamaño efectivos ${ }^{5}$, la ontología deleuziana, por su parte, busca estudiar procesos de individuación desde un punto de vista interno (sin apelar a categorías ni a ninguna forma general de representación previa), describiéndolos como movimientos de espacio-temporalización que dramatizan o actualizan una estructura inextensa, irrepresentable, diferencial o virtual, definida por una distribución de puntos singulares.

De acuerdo con lo desarrollado en Deleuze (1968a), este movimiento de actualización es conducido por factores individuantes llamados intensidades. La expresión es el acto por el cual esas intensidades individuantes desencadenan el proceso de actualización de lo virtual ${ }^{6}$. Nuestra tesis consiste en mostrar que este proceso expresivo es pensado por Deleuze como un movimiento - de corte topológico y morfogenético- de plegado, donde concurren una multiplicidad de tipos de pliegue, que interactúan mutuamente en la configuración de complejos espacio-temporales diversos, determinados por un trazado de frontera que constituye una interioridad topológicamente en contacto con un afuera. La interacción dinámica de esta topología adentro-afuera determina una individuación. Si, por un lado, los entes o individuos actuales (entes cualificados y extensos que habitan el espacio-tiempo) se manifiestan como los resultados de ese movimiento, por otro lado, ellos contienen elementos no actualizados en virtud de los cuales comunican con el afuera, y por el cual continúan plegándose de diversos modos, generando fronteras e individuaciones siempre otras. El pliegue constituye así un movimiento que define al Ser como Diferencia. Por todo esto, hablamos de una onto-topología deleuziana.

En lo que sigue, abordaremos la descripción de este proceso en cuatro momentos interrelacionados: 1) la concepción deleuziana del Ser unívoco

\footnotetext{
${ }^{5}$ Seguimos al matemático y filósofo Luciano Boi (1995 y 2011), que describe intuitivamente a la topología como "esa rama de las matemáticas donde la geometría es flexible" (2011, p. 23).

${ }^{6}$ Sobre la noción de expresión en Deleuze (1968 a y 1968b), remitimos a los cuidadosos análisis de Bowden (2017) y Ferreyra (2019). Pretendemos complementar aquí esas líneas de lectura, explicitando la dimensión matemática que estos comentadores no exploran. Esta explicitación nos permitirá posicionarnos en un tema de importante actualidad en los estudios deleuzianos, relativo a las relaciones entre lo virtual, lo actual y lo intensivo (al respecto, ver Clisby, 2016).
} 
que se expresa en cada individuación; 2) el campo pre-individual como lo expresado en un proceso de individuación; 3) el proceso de individuación mismo, como sistema expresante; 4) el concepto de expresión como torsión o pliegue inmanente entre el expresado (Idea virtual) y el expresante (intensidad). Seguiremos la vía de análisis de la individuación matemática, y señalaremos en nuestro recorrido las fuentes matemáticas deleuzianas que permiten encuadrar este proceso en toda su dimensión topológica.

\section{Univocidad del Ser y equivocidad del ente}

Según se asienta en el primer capítulo de Diferencia y repetición, existe una sola proposición ontológica: "el Ser es unívoco" (Deleuze, 1968a, p. 53). Lo designado en ella - el Ser- se dice en un solo y mismo sentido de todos sus designantes, o los elementos respecto a los cuales el Ser se predica - los entes-. Deleuze llama a estos designantes de diversas maneras: "modos numéricos" o "intrínsecos", "factores diferenciales", o "diferencias individuantes" (1968a, pp. 52-53). Mientras que cada ente puede ser designado a través de diferentes sentidos (como el "lucero de la mañana" y el "lucero de la noche", recordando el célebre ejemplo de Frege), y por lo tanto es equívoco, el Ser sólo puede decirse en un único sentido. Más aún, "lo esencial de la univocidad [...] [es que el Ser] se diga, en un solo y mismo sentido, de todas sus diferencias individuantes o modalidades intrínsecas" (Deleuze, 1968a, p. 53). Es decir que es tan necesario que su sentido sea unívoco como que este se exprese en diferentes individualidades equívocas. La univocidad se define entonces desde el carácter expresivo del Ser. El Ser se dice por igual de cada ente o individuo, y únicamente de ellos, pero cada uno de ellos es diferente. El Ser se dice entonces sólo de la diferencia en sí misma: este es el sentido del Ser ${ }^{7}$.

Ahora bien, esto exige que los límites que distinguen a un individuo de otro no sean parcelas preexistentes, fijas, impuestas desde el exterior. A causa de considerar al Ser como una suerte de superficie rígida que se divide en parcelas jerárquicamente organizadas — por ejemplo, a través de

${ }^{7}$ Si todos los individuos son distintos a pesar de expresar lo mismo en un mismo sentido, es porque aquello que expresan (el Ser) es en si mismo diferencia (ver Deleuze, 1968a, p. 57). Esta es la influencia fundamental de Nietzsche en la ontología deleuziana, a través de la cual se conduce una "inversión" del spinozismo (ver Ferreyra, 2019). Para una caracterización más completa del concepto deleuziano de univocidad tal como aquí lo comprendemos, ver Pachilla (2020). 
géneros y especies, o categorías, etc., que fungirían como coordenadas donde localizar los diferentes individuos-, el pensamiento representativo impone límites que rompen esa univocidad ontológica. El Ser no se diría por igual de cada ente, si a cada ente correspondiera una cierta "porción" o "parte" pre-definida de Ser (ser de tal género y de tal especie, ser tanto según la cantidad, tal según la cualidad, etc.). Deleuze propone en cambio que los límites de un individuo deben ser pensados a partir de su propia potencia intrínseca, como su capacidad de actuar y padecer, o, en un sentido más general, de variar. Cada individuo expresa en el mismo sentido el mismo Ser unívoco, pero no lo hace de la misma manera: cada uno lo hace en un cierto grado, según un cierto régimen de variación, definido por límites que encuadran su potencia de variar.

Esta concepción del límite implica una visión dinámica o procesual de la individuación, siendo un "individuo" todo aquello que posea una potencia de variar. Si esa potencia es llevada hasta el límite que la define, el individuo se excede a sí mismo, produce un "salto", una reconfiguración de límites. El límite, desde entonces, designa "aquello a partir de lo cual una cosa se despliega, y despliega toda su potencia [...]. Esta medida envolvente es la misma para todas las cosas, la misma para la substancia, la cualidad, la cantidad, etc., porque ella forma un solo máximo donde la diversidad desarrollada de todos los grados toca la igualdad que la envuelve." (Deleuze, 1968 a, p. 55; las cursivas son propias). Esta relación de envolvimiento y desenvolvimiento caracteriza la relación de los entes con el Ser -la diferencia ontológica一: el ente despliega la potencia envuelta en él dentro de ciertos límites, de modo tal que, al llevarla a su máximo, se vincula con esa medida envolvente común a todos los entes: el Ser unívoco, Diferencia, desmesura, hybris, devenir. Los individuos no son objetos que se distribuyen y clasifican en una cuadrícula prefijada del Ser, sino que el Ser entero se distribuye en la configuración dinámica de cada individuación, y sienta las bases de su exceso y su devenir hacia otra individuación.

Toda esta presentación ya implica una cierta tesitura topológica. La relación Ser-ente es planteada en términos espaciales. Construye una contraposición entre una caracterización extrínseca y estática de tipo continentecontenido (propia de la representación, que evoca una figura inmersa en un espacio ambiente de tipo euclidiano o cartesiano), y una caracterización intrínseca y dinámica de envolvimiento, desenvolvimiento y re-envolvimiento (propia de la filosofía de la diferencia, donde figura y espacio ambiente devienen indiscernibles en una dinámica morfogenética que motiva una producción múltiple de figuras heterogéneas). 
Pero esto deja planteados varios problemas: ¿por qué los “expresantes” habrían de desplegarse de modos distintos, si el Ser es para ellos igual y unívoco?, ¿qué determina el límite o la potencia propia de cada uno, aquello por lo cual se distingue del resto?, ¿cómo es efectuada o conducida a su límite esa potencia? Estas preguntas solo pueden abordarse desde la red de conceptos que Deleuze desarrolla en los capítulos IV y V de Diferencia y repetición, donde se juega el detalle de su apuesta ontológica. Allí, la Idea y la intensidad -o bien el campo pre-individual y el campo de individuación- forman, en unión inmanente, el "campo trascendental", la razón suficiente de todo ente o individuo constituido y representable. Cada uno de estos campos se define por ciertas dinámicas de plegado, sistematizadas y unificadas en la caracterización deleuziana de la expresión.

\section{Campo pre-individual: la multiplicidad perplicada}

¿De dónde proviene la equivocidad de los individuos, si el Ser que se dice de ellos es unívoco? La respuesta de Deleuze no carece de dificultades: el sentido expresado por los diferentes individuos, propiamente hablando, "no es". Las distinciones numéricas entre las modificaciones del Ser no responden a distinciones reales en él (así como en Spinoza los modos individuales no constituyen sustancias autónomas). Ahora bien, este "noser" de lo expresado, lejos de remitir a una forma de negación, carencia, defecto o privación de ser, remite a un "no-ser" por exceso, que insiste y persiste en -o en torno a- los entes. Se trata del campo virtual o pre-individual deleuziano (1968a, p. 89): “(no)-ser”, "extra-ser" o "?-ser", diferencial y problemático, que carece de existencia efectiva o actual, espacio-temporal, pero potencia la producción y determina la transformación de toda forma efectivamente existente. El sentido expresado por cada individuación, como momento equívoco del Ser unívoco, está definido por este punto extremo de la ontología, que intentaremos elucidar en esta sección. Deleuze lo presenta en el capítulo IV de Diferencia y repetición - "Síntesis ideal de la diferencia"-, bajo el concepto de Idea, en un recorrido interferido por numerosas nociones matemáticas.

En efecto, la matemática irrumpe desde los primeros momentos de la exposición de la Idea, caracterizada como un complejo de relaciones diferenciales $d y / d x$. Este tipo de relaciones permite mostrar el modo de funcionamiento del extra-ser problemático que genera los sentidos expresados por individuos diversos: se trata de una síntesis inmanente que pone en juego un momento en sí mismo indeterminado $(d x)$, un momento de determinación 
recíproca $(d y / d x)$ y un momento de determinación completa (valores singulares de $d y / d x)^{8}$. Estas figuras de la determinación definen los conceptos de límite y de potencia, que, como vimos, caracterizaban lo propio de cada individuo. Puesto que con esto se pretende indicar su génesis, el momento de la individualidad es aquí eliminado. Siguiendo a Jean Baptiste Bordas-Demoulin, Deleuze (1968a, p. 223) considera "lo individual" de una función matemática como constituido a la vez por su generalidad (la relación entre sus variables, cantidades abstractas de tipo $x, y$, etc.) y su particularidad (los valores numéricos de la función, obtenidos al asignar valores concretos a sus variables). Una función, en tanto individuo matemático, posee así una cualidad o especie (exponencial, polinómica trigonométrica, logarítmica, etc.), y cantidades o partes extensivas propias de esa especie (los valores concretos de la función o de su curva). La especie y las partes de la función permiten representarla en su modo o régimen de variación individual.

Bordas-Demoulin (1843, p. 457) afirma que la relación diferencial asociada a una función permite acceder a lo universal de ese individuo, universal que se halla "envuelto" en él. Llevar una función matemática a su universal equivale a conducirla al extremo de su potencia de variación, llevar su variación a su límite. Lo virtual, en tanto elemento diferencial indeterminado $(d x)$, remite a la potencia de un continuum que elimina la individualidad en favor de una pura variación evanescente, más allá de toda forma conceptual o intuitiva; dicho elemento no existe por sí, sino solo en una relación diferencial $(d y / d x)$, la cual, en tanto medio de determinación recíproca, genera una nueva individualidad que revela lo universal de la anterior: una función derivada (así, el continuum diferencial, al llevar una variación a su límite, constituye necesariamente un nuevo límite). Mediante ese nuevo individuo matemático (derivada) es posible mapear los puntos singulares que determinan la estructura global del anterior (función primitiva); de ahí que actúe como universal. Pero eso universal no subsiste por sí mismo, sino en el movimiento de disolución, génesis y enlace entre individualidades diversas.

Bajo influencia de Albert Lautman (1938, pp. 130-9), Deleuze menciona los puntos singulares definidos por Poincaré: cuellos, nudos, focos y centros (Deleuze, 1968a, p. 230; y 1969, p. 127). Ellos son distintos tipos de accidentes topológicos de una curva matemática, que determinan

\footnotetext{
${ }^{8}$ Nos hemos detenido extensamente acerca del sentido y las fuentes de este movimiento filosófico deleuziano en Santaya (2017), por lo que aquí nos limitaremos a señalar lo esencial en vistas al argumento presente.
} 
cualitativamente su espacio (previamente al cálculo cuantitativo). La Idea deleuziana es, pues, el lugar de la génesis del sentido, del mismo modo en que la relación diferencial es el lugar de la génesis de singularidades. En la Idea, o campo pre-individual, coexisten inmediatamente lo más universal (el elemento diferencial que suprime el momento individual) con lo más singular (los puntos que estructuran el espacio de una individuación). Para emitir y distribuir estas singularidades, la Idea opera una conexión entre funciones individuales diversas. En este sentido, ella es el sitio de un "cambio de función” (Deleuze, 1968a, p. 224; Bordas-Demoulin, 1843, p. 471), y a la vez, la pura potencia de generar nuevas funciones. El campo pre-individual, con sus singularidades, es el "elemento puro de la potencialidad" (Deleuze, 1968a, p. 227) ${ }^{9}$.

La noción de Idea alcanza su punto de mayor resonancia topológica con la definición, de inspiración riemanniana, que Deleuze presenta luego de estos desarrollos: "Una Idea es una multiplicidad definida y continua de n-dimensiones" (Deleuze, 1968a, p. 236). Con esta remisión a la geometría de Riemann (1898), se define el ámbito de lo virtual como red de relaciones recíprocas entre elementos diferenciales que conforman una suerte de topología de hiper-superficies. La compleja estructura no-euclidiana de una multiplicidad riemanniana permite construir progresivamente su espacio de un modo intrínseco o local. Al asignar funciones que especifican las partes de la multiplicidad y sus conexiones con las partes restantes, determina su métrica (es decir, el patrón de comparación entre las formas y figuras que pueden representarse en ese espacio). Más allá de las métricas toleradas por una multiplicidad, que permiten individuar en ella distintas figuras, los elementos diferenciales subyacen como fondo problemático, no individuado ni individuable: el continuum de lo virtual, con sus relaciones y distinciones, conforma un plano de absoluta coexistencia, en un estado que Deleuze llama perplicación: "Las Ideas son complejos de coexistencia, todas las Ideas coexisten de una cierta manera. [...] Las Ideas, las distinciones de Ideas, no son separables de sus tipos de variedad y de la manera en que cada uno penetra en los otros. Proponemos el nombre de perplicación para designar ese estado distintivo y coexistente de la Idea" (Deleuze, 1968a,

${ }^{9} \mathrm{La}$ diferencia de potencias entre variables es fundamental, porque es a partir de ella que se pliega el espacio en una curva, y porque posibilita la derivabilidad de una función. Deleuze parece seguir implícitamente a Hegel (2013, p. 319) en este punto, cosa explicitada en Deleuze y Guattari (1991, p. 115, donde se usan los mismos ejemplos de funciones matemáticas). Veremos en las próximas secciones la importancia de la potencia en toda la trama ontológica. 
pp. 241-2). Se trata del tipo de pliegue correspondiente a la multiplicidad o variedad virtual (retomaremos esto en las secciones siguientes).

Ese fondo de coexistencia de relaciones y singularidades perplicadas debe ser entonces individuado mediante las funciones que habilitan una representación espacial efectiva. El desarrollo de la Idea como multiplicidad diferencial no provee los elementos para pensar este proceso. Sugerentemente, el propio Riemann, al finalizar la disertación en la que introduce su noción de multiplicidad, dice que la determinación de las funciones correspondientes a la naturaleza del espacio empírico exige "que el fundamento de las relaciones métricas sea buscado fuera de él, en las fuerzas de ligazón que actúan en él" (Riemann, 1898, p. 297) ${ }^{10}$. Hay, en este sentido, una preeminencia de la fuerza física como factor fundamental para construir las propiedades que permiten representar el espacio empírico, dándole carnadura a la multiplicidad en tanto pura estructura diferencial. En términos deleuzianos, las determinaciones del ámbito virtual, dadas por las singularidades, requieren de intensidades que produzcan los espacio-tiempos correspondientes a esas singularidades. Esto nos conduce del campo pre-individual al campo de individuación.

\section{Campo de individuación: la multiplicidad implicada-explicada}

Como vimos, Deleuze llamaba a los factores que producían la diversificación del Ser tanto "diferenciales" como "individuantes". En el desarrollo de las nociones que detallan el movimiento ontológico, estos factores pasan a distinguirse con precisión. Venimos de describir el campo pre-individual como el ámbito perplicado de factores diferenciales que, en tanto (no)-ser o extra-ser, estructuran o determinan el sentido expresado por un ente individual. Los factores individuantes, por su parte, remiten a cómo ese sentido es efectivamente expresado a través de la intensidad — proceso desarrollado en el capítulo V de Diferencia y repetición, "Síntesis asimétrica de lo sensible"- Deleuze remite la dinámica energética de una diferencia de potencial física (bajo el modelo de los principios causales de disimetría en termodinámica y cristalografía, desarrollados, respectivamente, por Carnot y

${ }^{10}$ Como afirma Boi (1995, p. 164): “a cada elección de la función que determina la métrica corresponde un espacio diferente que caracteriza la variedad. Así, el concepto de espacio se encuentra no solamente aclarado, sino especificado y relativizado por el de métrica". Esto convierte a la multiplicidad en un campo de experimentación, una matriz de coexistencia de diversos espacios posibles. 
Curie $)^{11}$ para construir el concepto de campo de individuación, complemento inseparable del campo pre-individual. La noción de asimetría, en tanto caracteriza una diferencia de potencial, excede su origen geométrico para describir un desequilibrio físico que tiende a una situación de equilibrio. Según la concepción de Simondon (2009), que Deleuze retoma, esa tendencia es condición necesaria para un proceso de individuación cualquiera.

El campo de individuación es pues una sede de asimetrías que dan pie a esas "fuerzas de ligazón" que — como quería Riemann— permiten la construcción de la métrica efectiva de la estructura topológica de la multiplicidad. En este sentido, la individuación intensiva efectúa la estructura virtual, produce un dinamismo espacio-temporal correspondiente a las determinaciones del campo pre-individual. Deleuze (1968a, p. 315) tiene esto en mente cuando comienza a describir la interacción entre ambos campos: ella supone "dos tipos de relación, relaciones diferenciales en la síntesis recíproca de la Idea, relaciones de intensidad en la síntesis asimétrica de lo sensible. La síntesis recíproca $d y / d x$ se prolonga en la síntesis asimétrica que liga $x$ a $y$. El factor intensivo es una derivada parcial, o la diferencial de una función compuesta". El "factor intensivo" se expresa matemáticamente como una función, entendida como sintesis asimétrica entre variables independientes.

A la hora de seguir el hilo topológico de la ontología, se destaca en el pasaje recién citado la noción de "derivada parcial", que consiste en derivar una parte de una función primitiva de múltiples variables independientes, o más precisamente, diferenciar dicha función con respecto a una de esas variables (una de sus asimetrías), suponiendo a las restantes como constantes. Las derivadas parciales se aplican a un amplio rango de fenómenos físicos (propagación del sonido y el calor, elasticidad, dinámica de fluidos, electromecánica, mecánica cuántica, entre otros). Se trata de una herramienta fundamental para describir esas "fuerzas de ligazón" referidas por Riemann: la noción de derivada covariante, que es una generalización de la de derivada parcial a espacios $n$-dimensionales, permite pasar de las coordenadas rectilíneas — propias del análisis tradicional- a coordenadas curvilíneas, propias de las multiplicidades de Riemann. Entre otras aplicaciones, esto permite analizar las posibilidades de traslación paralela de vectores a través de una multiplicidad de curvatura y métrica variables, efectuando diversas conexiones posibles entre sus vecindades diferenciales, es decir, efectuando

${ }^{11}$ Ver McNamara (2016) para un desarrollo del sentido ontológico de las fuentes físicas que concurren en la descripción de la síntesis asimétrica de la intensidad deleuziana. 
la estructura topológica de la variedad en una cierta configuración espacial representable ${ }^{12}$.

Los elementos diferenciales indeterminados carecían por sí mismos de representación, así como los puntos singulares (que la estructura virtual sólo permitía descubrir y localizar). El espacio topológico se efectúa, se representa o se individúa en una forma extensiva a través de las funciones que describen las líneas que constituyen y conectan sus regiones: "La individuación es el acto de la intensidad que determina a las relaciones diferenciales a actualizarse, siguiendo líneas de diferenciación, en las cualidades y extensiones que ella crea" (Deleuze, 1968, pp. 316-317). La intensidad, en tanto función derivada parcial que integra localmente una parte de la multiplicidad virtual, crea un espacio extenso y cualificado en correspondencia con la matriz diferencial e inextensa, y de ese modo determina a las determinaciones virtuales a actualizarse en las cualidades y extensiones, o las especies y partes, que constituyen la generalidad y la particularidad de un individuo. En la sección previa indicamos cómo esas especies y partes remiten siempre a otras especies y partes, pues la Idea se constituye como universal de un individuo enlazándolo necesariamente a otra individualidad (como era el caso, descripto por Bordas-Demoulin, entre primitiva y derivada, relación diferencial mediante). Se produce así una remisión indefinida - mediada por las relaciones virtuales - de individuo a individuo, entre individualidades mutuamente envueltas unas en otras, donde las relaciones diferenciales muestran la razón o el orden de ese envolvimiento. De hecho, una variedad riemanniana es por definición infinitamente diferenciable o derivable: todas sus funciones "envuelven" su diferencial, como universal pre-individual que las remite a otras funciones. Así concebido, un proceso de individuación puede descomponerse en sub-individuaciones, como también remitirse a supra-individuaciones en las que se acopla.

Este juego entre relaciones diferenciales-ideales y asimétricas-intensivas manifiesta la distinción entre dos tipos de multiplicidades. "Las Ideas son multiplicidades virtuales, problemáticas o "perplejos», hechas de relaciones entre elementos diferenciales. Las intensidades son multiplicidades implicadas, «implejos», hechas de relaciones entre elementos asimétricos que

${ }^{12}$ En su descripción de los espacios de Riemann, Lautman (1938, pp. 45-48) otorga una gran importancia a este transporte de vectores paralelos, en un pasaje recuperado ulteriormente por Deleuze y Guattari (1980, p. 462). Sobre la noción de derivada covariante ver Kline (1992, pp. 1488 y ss.). Puede verse también Jedrzejewzki (2017, especialmente p. 315316) para más detalles técnicos acerca de cómo el espacio amorfo de la pura multiplicidad deviene una estructura actual mensurable. 
dirigen el curso de actualización de las Ideas [...] la potencia de la intensidad (profundidad) está fundada en la potencialidad de la Idea" (Deleuze, 1968a, p. 315). La implicación aparece entonces como un nuevo tipo de multiplicidad, que debe pensarse estrictamente en sus relaciones con la multiplicidad ideal perplicada. Mientras que esta se caracterizaba por la absoluta coexistencia de los elementos diferenciales y singularidades en mutua interpenetración, aquella aparece como el agente del movimiento de envolvimiento-desenvolvimiento presente en cada individuación, motivado por la síntesis asimétrica de una diferencia de potencial intensivo, o como lo llama aquí Deleuze, "profundidad". La potencia de esta profundidad-que, como indica el pasaje citado, está "fundada" en la potencialidad virtualsupone una carga envuelta que tiende por su propia fuerza a desenvolverse. En tanto supone a la vez relaciones de envolvimiento y desenvolvimiento, la implicación en profundidad goza de un doble aspecto (1968a, p. 309): uno secundario por el cual la potencia intensiva se explica, o se despliega en cualidades y cantidades extensas (figuras estables que obedecen a una métrica como patrón de representación del individuo acabado), y otro primario por el cual esa potencia permanece implicada en sí misma. Según este aspecto primario, todo proceso de explicación supone un resto de potencial no agotado, en comunicación con un puro campo intensivo que Deleuze llama - tomando un concepto leibniziano- spatium, poblado de asimetrías no explicadas o "distancias". Esto supone una nueva distinción a partir de la multiplicidad intensiva:

Debemos, desde entonces, distinguir dos tipos de multiplicidades, como las distancias y las longitudes: multiplicidades implícitas y las explícitas, aquellas cuya métrica varía con la división y aquellas que llevan consigo el principio invariable de su métrica. Diferencia, distancia, desigualdad, son los caracteres positivos de la profundidad como spatium intensivo. Y el movimiento de la explicación es aquél por el cual la diferencia tiende a anularse, pero también las distancias a extenderse, a desarrollarse en longitudes, y lo divisible a igualarse. (Deleuze, 1968a, pp. 306-307).

Se definen así los dos tipos de pliegue, copresentes y complementarios, del campo de individuación: implicación y explicación. La producción de individuos actuales, compuestos de cualidades y partes extensivas, supone la explicación de la potencia implicada en una profundidad o campo de individuación intensivo definido por una asimetría. Esa explicación "anula" una diferencia, agota un potencial, pues traduce su espacio esencialmente asimétrico en una configuración métrica compuesta de partes homogéneas o longitudes. El espacio asimétrico, considerado en sí mismo, excede toda explicación, pues consiste en puras heterogeneidades: no ya longitudes (que 
se dividen en partes homogéneas al infinito) sino distancias implicadas o envueltas en un spatium intensivo, inagotable e inexplicable, que no cesa de dividirse y detenta "la potencia de dividirse cambiando de naturaleza" (Deleuze, 1968a, p. 326) ${ }^{13}$.

La cuestión de la articulación entre los tres tipos de multiplicidad descriptos hasta aquí - perplicadas, implícitas y explícitas - se completa con la descripción sobre el modo en que la individuación desencadena la actualización de las Ideas. El concepto de expresión sistematiza esa articulación compleja, y nos reenvía a la cuestión del Ser unívoco.

\section{La torsión expresiva: (des)pliegues sobre (re)pliegues}

La definición técnica de la expresión contiene a su vez resonancias topológicas: "Por expresión entendemos, como siempre, esta relación que comporta esencialmente una torsión entre un expresante y un expresado, tal que lo expresado no existe fuera del expresante, aunque el expresante se relacione con aquél como con algo completamente otro" (Deleuze, 1968a, p. 334). Esto es una paradoja: lo expresado (la Idea) no existe por fuera de su expresión (la relación de torsión) que la vuelve dependiente del expresante (la intensidad) ${ }^{14}$. Veremos que se trata de una paradoja tanto temporal como espacial —esta última nos interesa especialmente- Tanto lo expresado como el expresante poseen en sí mismos una estructura articulada en diversos niveles, lo que hace de la expresión una noción de gran complejidad.

${ }^{13}$ Los conceptos de spatium y distancia (como sede de puras relaciones asimétricas, previas a su traducción en longitudes) son tomados de Leibniz — por mediación de Gueroult (2017) - y precisados a través Russell (1899), en su recuperación de Meinong. Ver Deleuze (1968a, p. 306); ver también Jedrzejewzki (2017, p. 318) para una distinción entre distancias intensivas y longitudes extensivas.

${ }^{14}$ La expresión como paradoja articula la lectura deleuziana de Spinoza (1968b, p. 310): “Ahora bien, la paradoja es que, a la vez, lo «expresado» no existe por fuera de la expresión, y sin embargo no se le asemeja, pero está esencialmente relacionado a aquello que se expresa, como distinto de la expresión misma". La expresión inmanente resuena desde la tradición renancentista con el concepto de pliegue: "La inmanencia se define por el conjunto de la complicación y de la explicación [...]. Las cosas permanecen inherentes al Dios que las complica, como Dios permanece implicado por las cosas que lo explican. [...] La expresión comprende todos estos aspectos: complicación, explicación, inherencia, implicación" (Deleuze, 1968b, p. 159). La recuperación deleuziana de estos conceptos para pensar la expresión añadirá dos figuras del pliegue propias de su ontología (perplicación y replicación), que analizaremos en esta sección. 
La expresión es en primer lugar temporalmente paradójica. La potencia de la intensidad, fundada sobre la potencialidad de la Idea, efectúa esa potencialidad expresándola, en un proceso fuera del cual lo expresado (la Idea) no existe; simultáneamente, las relaciones y singularidades de la Idea expresada determinan el sentido de su expresión, dada por la intensidad. Si la Idea determina cómo una actualización se desarrolla, la intensidad determina el hecho de que haya actualización. La intensidad selecciona y conduce — como derivada parcial — una parte de lo virtual hacia lo actual; pero antes de la actualización no hay una Idea ya hecha esperando ser espacializada: el acto de expresión hace coexistir, en su torsión, lo expresante y lo expresado. Lo expresado surge a la vez que funda la potencia de aquello que lo constituye al expresarlo, emitiendo las singularidades o repartiendo los potenciales por los cuales la profundidad intensiva se configura y se explica en cualidades y extensiones actuales.

Ahora bien, así como las Ideas remitían unas a otras en perplicación, las intensidades lo hacen en implicación; ¿cómo se da la explicación de tan solo una parte de esta maraña caótica? Sucede que existen dos niveles presentes en cada expresión, claro y confuso, basados en la naturaleza intensiva del expresante (envolvente-envuelto):

He aquí que la intensidad, la diferencia en sí misma, expresa las relaciones diferenciales y los puntos remarcables correspondientes. Ella introduce entre estas relaciones, y entre las Ideas, un nuevo tipo de distinción. Ahora las Ideas, las relaciones, las variaciones de estas relaciones, los puntos remarcables, son de algún modo separados: en lugar de coexistir, ellos entran en relaciones de simultaneidad y sucesión. Sin embargo, todas las intensidades están implicadas las unas en las otras, cada una siendo a su vez envolvente y envuelta. En consecuencia, cada una continúa expresando la totalidad cambiante de las Ideas, el conjunto variable de las relaciones diferenciales. Pero ella no expresa claramente sino algunas, o algunos grados de variación. Las que expresa claramente son precisamente aquellas a las que ella apunta directamente en su función envolvente. No por eso ella deja de expresar todas las relaciones, todos los grados, todos los puntos, pero confusamente, en su función envuelta. [...] Las intensidades envolventes (la profundidad) constituyen el campo de individuación, las diferencias individuantes. Las intensidades envueltas (las distancias) constituyen las diferencias individuales. (Deleuze, 1968a, pp. 325-326).

Los dos aspectos de la implicación constituyen los polos de una oscilación intensiva, un vaivén de la intensidad, que realiza en cada expresión una torsión en la cual ella vuelve una cara hacia las extensiones y cualidades (la expresión clara de una Idea a través del aspecto explicado de la profundidad), y otra cara hacia el todo de lo virtual (la expresión confusa de las Ideas por el aspecto implicado del spatium). Si lo individual —recuperando una vez más la expresión de Bordas-Demoulin-envuelve lo universal, es 
porque está en relaciones de envolvimiento con todas las intensidades. Siendo cada intensidad la expresión clara de una determinación pre-individual, y estando cada Idea en estado de coexistencia perplicada con la totalidad del campo virtual, todas las Ideas están expresadas en cada individuo. Pero no lo están del mismo modo: cada intensidad reordena de un modo diferente el conjunto de las Ideas expresadas de acuerdo a sus esferas clara y confusa ${ }^{15}$. En virtud de este reordenamiento, las Ideas mismas se modifican constantemente: el spatium implicado no cesa de dividirse cambiando de naturaleza, "aclarando" otras y otras singularidades, emitidas en el continuum preindividual perplicado por obra de las intensidades individuantes. Las diferentes expresiones claras, efectuadas por factores intensivos, homogeneizan una cierta asimetría a la vez que la comunican con una profundidad caótica inagotable, que expresa confusamente una emisión de singularidades siempre otras, nuevos potenciales que fundarán a su vez nuevas asimetrías, que aclararán otras singularidades... En este sentido, la "totalidad" propia de lo virtual y de lo intensivo, lejos de remitir a un conjunto o estructura cerrada sobre sí, es aquello que impide la clausura: ella abre cada individuo a un afuera en el cual este se disuelve y reconfigura sus potenciales, sus límites. El (no)-ser problemático es una emisión de las diferencias virtuales que insisten y persisten en torno a los productos de toda síntesis asimétrica, motorizando y enlazando nuevas asimetrías o campos intensivos.

Este vínculo entre esferas clara y confusa muestra la paradoja espacial de la expresión: ellas conforman un fenómeno de frontera donde todo el adentro está topológicamente en contacto con todo el afuera. Las líneas que actualizan un virtual se producen por explicación de una profundidad que envuelve la totalidad de las series intensivas según un cierto orden, dado por las singularidades y relaciones expresadas claramente en ese envolvimiento. Se constituye así una peculiar espacialidad donde cada parte contiene dentro de si al todo. Cada individuo se distingue por el modo en que, desplegando sus partes extensivas, pliega la totalidad de las intensidades envueltas que remiten a las Ideas confusamente expresadas en esos pliegues.

Un pequeño excursus lingüístico puede ser clarificador en este punto. Debemos dar toda la importancia al morfema "pli", que se repite en todos los niveles de la torsión expresiva (perplicación, implicación, explicación.),

${ }^{15}$ Más allá de la influencia spinozista respecto a la noción de expresión, Deleuze (1968a, p. 326) recupera aquí a Leibniz, siguiendo el ejemplo de la percepción del ruido del mar a partir de una síntesis de pequeñas percepciones, que distingue un orden de expresión claro y uno confuso: el ruido actual de la ola y el murmullo diferencial de la totalidad, remitiendo con esto a la relación "mundo-mónada". 
y analizar de qué modo ese morfema aparece resignificado con cada uno de sus prefijos. De origen latino, "per-", "im-" y "ex-", significan, respectivamente, "a través de" (o también "totalmente"), "hacia adentro", y "hacia afuera" ". A estos términos, Deleuze suma otras dos figuras del pliegue, con menor presencia textual, pero con no menor importancia ontológica: la complicación y la replicación. "Com-" significa "reunión", "todo junto", mientras que "re-", "repetición”, "volver hacia atrás". (Estos cinco "pliegues" aparecen enumerados y definidos en Deleuze, 1968a, pp. 359-360.)

La implicación consiste en un plegar hacia adentro, constituyendo una interioridad de la cual la intensidad es simultáneamente sujeto y objeto: envolvente y envuelta. En tanto envolvente, ella es una profundidad que interioriza un potencial por el cual expresa claramente ciertas relaciones y puntos virtuales, actualizándolos por su explicación en especies y partes extensivas. Esta explicación constituye una suerte de movimiento de plegado hacia afuera, despliegue, por el cual la profundidad crea cualidades y extensiones en una puesta "fuera de sí", "recubriendo" ${ }^{17}$ la asimetría con longitudes homogéneas. A pesar de este recubrimiento, la interioridad remite a todas las intensidades, la multiplicidad implícita que expresa confusamente todas las Ideas. Este estado del pliegue es el de la complicación: la reunión de todas las intensidades juntas, "el estado del caos que retiene y comprende a todas las series intensivas actuales que corresponden con las series ideales, que las encarnan y afirman su divergencia" (Deleuze, 1968a, p. 359). Así, la profundidad realiza un (des)pliegue que exterioriza su potencia productiva en una extensión cualificada, partes extra partes, pero hacia adentro remite al caos de las distancias del spatium "que envuelve todos sus grados en la producción de cada uno” (Deleuze, 1968a, p. 310).

La perplicación de las Ideas, a su vez, puede definirse — siguiendo los sentidos indicados del prefijo "per-" - como lo "totalmente" plegado y como un "a través" del pliegue: en tanto lo totalmente plegado, el ámbito de lo virtual coexiste en su totalidad consigo mismo, es inmediatamente co-presente a sí mismo en todos sus puntos y relaciones, que penetran unas en las otras: velocidad infinita, continuum donde todo emerge y se funde inmediatamente, sin separación, sin distancia. Si está totalmente plegada, plegada de todos los modos a la vez, entonces no lo está de ninguno: es la

${ }^{16}$ Todo esto puede verse para el sentido de los prefijos latinos, pero también para el francés y el español, donde mantienen un mismo sentido. Remitimos a los siguientes diccionarios en línea: https://fr.wiktionary.org/wiki/Annexe:Pr\%C3\%A9fixes_en_fran\%C3\%A7ais\#I (francés); http://etimologias.dechile.net/latin/ (latín). Acceso el 5/8/2020.

${ }^{17}$ Esta es terminología del propio Deleuze (ver 1968a, pp. 288, 294 y 305). 
implicación individuante — profundidad—quien establece separaciones en ese caos, plegándolo según un cierto orden. Cada implicación es un pliegue, un doblez que articula un individuo actual con su campo pre-individual. Pero este campo pasa a través del pliegue: excede la implicación intensiva hacia un afuera trascendental, comunicando con otros pliegues intensivos, (re)generando potenciales y replegando el conjunto.

Así, en cada individuo, el adentro implicado del spatium comunica inmediatamente con el afuera perplicado de lo virtual. Las distancias implicadas que una profundidad envuelve, y las Ideas confusamente expresadas por ellas, constituyen la frontera topológica que se expresa en cada forma actual explicada. La expresión es paradójica porque es a la vez lo que constituye y lo que rompe la interioridad, minando desde su adentro el proceso de individuación e impulsándolo al afuera, reconfigurando la frontera. Esta dinámica excede totalmente la lógica de la representación, del mismo modo en que ciertos objetos topológicos, como la botella de Klein, remiten a un espacio paradójico, absoluto, auto-contenido en sí mismo, no orientable, y cuya articulación intrínseca depende de dimensiones que superan la intuición empírica ${ }^{18}$.

La apertura a ese afuera trascendental está conceptualizada bajo la figura de la replicación, como el elemento que, en el contexto de un sistema actual (cualitativo-extensivo) en vías de explicación, "testimonia aún la persistencia de los problemas" (Deleuze, 1968a, p. 360). La persistencia de los problemas es otra aparición destacable del prefijo "per-": ellos continúan operando a través de sus distintas soluciones. En el seno de un individuo extenso constituido, la replicación abre la posibilidad de un nuevo ascenso del fondo problemático virtual. El prefijo "re-" indica la Repetición (repetición del pliegue, y por lo tanto de la expresión); se trata nada menos que del retorno de la Diferencia: la emergencia, en lo extensivo, del sin-fondo intensivo-virtual que reorganiza la torsión expresiva. Dicha torsión compone así un movimiento complejo que supone la interacción entre todos los niveles de la multiplicidad, particularmente entre el ser implicado en profundidad y el (no)-ser perplicado, Ser y extra-ser.

${ }^{18}$ Sobre la botella de Klein, ver Boi (2011, pp. 40-45). Este objeto topológico puede definirse al "pegar" dos cintas de Möbius por sus líneas de frontera. Dicha cinta es la imagen utilizada por Deleuze $(1969$, p. 149) para pensar la operación del sentido por la cual se establece la conexión entre la profundidad de los cuerpos y la superficie metafísica poblada de singularidades, en términos de una continuidad entre un anverso y su reverso. 


\section{Conclusiones}

Si bien la ontología no se basa directamente sobre la topología, de modo que pueda plantearse un "diccionario" que haga corresponder mutuamente sus términos, hemos constatado que la matemática guarda un potencial expresivo caro a Deleuze. La dinámica ontológica de la "frontera", descripta de la mano de la topología, permite abordar la tesis ontológica fundamental de Diferencia y repetición, la del Ser como devenir en el Eterno Retorno. Volviendo a la proposición ontológica: "el Ser es unívoco", y lo es porque sólo se dice de lo que difiere, de la diferencia en sí misma. "La univocidad del ser y la diferencia individuante [la profundidad] tienen un lazo, fuera de la representación, tan profundo como el que la diferencia genérica y la diferencia específica tienen en la representación desde el punto de vista de la analogía" (Deleuze, 1968a, p. 388). Más allá de la lógica euclidiana de la representación, la expresión intensiva traza la frontera propia a cada pliegue, donde el Ser entero se distribuye en la profundidad de sus diversos modos intrínsecos. "De la intensidad a la profundidad se anuda la más extraña alianza, la del Ser consigo mismo en la diferencia [...]. En el ser, la profundidad y la intensidad son lo Mismo, pero lo mismo que se dice de la diferencia" (Deleuze, 1968a, pp. 297-298). La intensidad es el Eterno Retorno, el corazón del Ser; pero un corazón animado por movimientos de sístole y de diástole que suponen puntos estructurantes, singularidades del (no)-ser que refundan y transforman permanentemente su potencia, modificando los pliegues que reorientan, distribuyen y redistribuyen en cada expresión al Ser entero. Se repite así la potencia de la diferencia, en la dinámica inmanente de un paradójico espacio auto-contenido y no-orientable, que desborda sus configuraciones fortuitas y genera permanentemente otras, plegándose, desplegándose y replegándose de infinitos modos en cada una.

\section{Bibliografía}

Badiou, A., El ser y el acontecimiento, trad. Cerdeiras, R., Cerletti A., y Prados, N., Buenos Aires, Manantial, 2007.

Boi, L., Le problème mathématique de l'espace. Une quête de l'intelligible, Berlín, Springer, 1995.

Boi, L., Morphologie de l'invisible, Limoges, Presses Universitaires de Limoges, 2011.

Bordas-Demoulin, J. B., Le cartésianisme ou la véritable rénovation des sciences, París, J. Hetzel Libraire-éditeur, 1843. 
Bowden, S., "The Intensive Expression of the Virtual: Revisiting the Relation of Expression in Difference and repetition", Deleuze \& Guattari Studies (2017), 11(2), 216-239.

Burchill, L., "The Topology of Deleuze's Spatium", Philosophy Today (2007), 51, 154-160.

Calamari, M., "Riemann-Weyl in Deleuze's Bergsonism and the Constitution of the Contemporary Physico-Mathematical Space”, Deleuze Studies (2015), 9(1), 59-87.

Clisby, D., “¿El dualismo secreto de Deleuze? Versiones en disputa sobre la relación entre lo virtual y lo actual”, trad. Pachilla, P., Ideas. Revista de filosofía moderna y contemporánea (2016), 4, 120-148.

DeLanda, M., Intensive Science and Virtual Philosophy, Londres/Nueva York, Continuum, 2002.

Deleuze, G., Nietzsche et la philosophie, París, PUF, 1962.

Deleuze, G., Différence et répétition, París, PUF, 1968a.

Deleuze, G., Spinoza et le problème de l'expression, París, PUF, 1968b.

Deleuze, G., Logique su sens, París, Minuit, 1969.

Deleuze, G., Foucault, París, Minuit, 1986.

Deleuze, G., Le pli. Leibniz et le baroque, París, Minuit, 1988.

Deleuze, G., Pourparlers, París, Minuit, 1990.

Deleuze, G., L'île déserte et autres textes. Textes et entretiens 1953-1974, París, Minuit, 2002.

Deleuze, G. y Guattari, F., Mille Plateaux. Capitalisme et schizophrénie 2, París, Minuit, 1980.

Deleuze, G. y Guattari, F., Qu'est-ce que la philosophie?, París, Minuit, 1991.

Deleuze, G. y Parnet, C., Dialogues, París, Flammarion, 1996.

Duffy, S., Deleuze and the History of Mathematics. In Defense of the New, Edimburgo, Edinburgh University Press, 2013.

Ferreyra, J., "Deleuze y el problema de la expresión”, Cadernos Espinosianos (2019), 41, 137-172.

Gueroult, M. "El espacio, el punto y el vacío en Leibinz", trad. Victoria Rusconi, en: Mc Namara, R., y Santaya, G. (eds.): Deleuze y las fuentes de su filosofía V, Buenos Aires, RAGIF Ediciones, 2017, pp. 251-282.

Hegel, G. W. F., Ciencia de la lógica, trad. Rodolfo Mondolfo, Buenos Aires, Las cuarenta, 2013.

Jedrzejewzki, F., "Deleuze et la géométrie riemannienne: une topologie des multiplicités", en: Ji, L., Papadopoulos, A., y Yamada, S. (eds.): From Riemann to Differential Geometry and Relativity, Cham, Springer International Publishing, 2017, pp. 311-328. 
Kline, M., Historia del pensamiento matemático desde la antigüedad hasta nuestros días, trad. Martínez, M., Tarrés, J. y Casal, A., Madrid, Alianza, 1992.

Lautman, A., Essai sur les notions de structure et d'existence en mathématiques, París, Hermann, 1938.

Mc Namara, R., "Degradación empírica y repetición trascendental”, en: Ferreyra, J. (ed.): Intensidades deleuzianas. Deleuze y las fuentes de su filosofía III, Buenos Aires, La Cebra, 2016, pp. 67-84.

Pachilla, P., "Univocidad", en Ferreyra, J. y Soich, M., Introducción en Diferencia y repetición, Buenos Aires, RAGIF Ediciones, 2020, pp. 21-31.

Plotnitsky, A., "Manifolds: On the concept of space in Riemann and Deleuze», en: Duffy, S. (ed.): Virtual Mathematics. The logic of Difference, Londres, Clinamen Press, 2006, pp. 187-208.

Riemann, B., "Sur les hypothèses qui servent de fondement à la géométrie", trad. francesa de Jules Houël, en: B. Riemann, Euvres mathématiques, París, Gauthier-Vilars, 1898, pp. 280-299.

Russell, B., «Review of Meinong», Mind (1899), 8.30, 251-256.

Santaya, G., El cálculo trascendental. Gilles Deleuze y el cálculo diferencial: ontología e historia, Buenos Aires, RAGIF Ediciones, 2017.

Santaya, G., "Sobre la topología conceptual como práctica de la ontología”, en Heffesse, S., Pachilla, P. y Schoenle, A., Lo que fuerza a pensar. Deleuze: ontología práctica, Buenos Aires, RAGIF Ediciones, 2019, pp. 45-56.

Salanskis, J. M., «Ideas and Destination», en : Patton, P. (ed.), Deleuze. A Critical Reader, Oxford, Blackwell, 1996, pp. 57-80.

Simondon, G., La individuación a la luz de las nociones de forma y de información, trad. Ires, P., Buenos Aires, Cactus - La Cebra, 2009.

Widder, N., "The mathematics of continuous multiplicities: The Role of Riemann in Deleuze's Reading of Bergson", Deleuze \& Guattari Studies (2019), 13(3), 331-354.

Zalamea, F., "Diferencia y repetición: preludios en la matemática moderna y ecos en la matemática contemporánea", Universitas Philosophica (2020), 37(74), 139-153. 